\title{
Desafiando el contexto: Apuntes a los debates contemporáneos sobre redes y escalas
}

\author{
Challenging context: Notes to the contemporary debates about \\ networks and scales
}

\author{
Juan Camilo Perdomo-Marín \\ Universidad de Caldas (Colombia) \\ juancaperdo@hotmail.com (COLOMBIA)
}

Recibido: 21.092018

Aceptado: 13.12.2019

\section{RESUMEN}

El presente escrito sintetiza, evalúa y amplia críticamente los debates contemporáneos sobre el ejercicio de contextualización para comprender los nuevos desafíos y posibilidades que acarrea la investigación social. Para esta tarea, primero, se introducirán las ideas centrales de los Estudios Sociales de la Ciencia y la Tecnología con la finalidad de comprender cómo el proceso de contextualización afecta directamente el objeto de estudio. Segundo, se señalará que este objeto posee un estatus ontológico emergente puesto que se deben redefinir constantemente las relaciones que lo componen. Tercero, se resaltarán las contribuciones de Marilyn Strathern a los debates anteriores por medio de sus reflexiones sobre las escalas. Por último, mediante los conceptos de lógicaperceptiva, hacer-escala y mereología se sustentará que la ontología, la epistemología, la metodología y la reflexividad se encuentran en una retroalimentación permanente. Además, se resaltará que este vínculo implica reevaluar la posición política del investigador, la forma en que se entiende el trabajo de campo y el mundo no-humano.

\section{PALABRAS CLAVE}

Contexto, ontología, reflexividad, Estudios Sociales de la Ciencia y la Tecnología, Marilyn Strathern. 


\begin{abstract}
This paper synthesizes, evaluates and critically broadens contemporary debates about the exercise of contextualization to understand the new challenges and possibilities that social research brings. For this task, first, the central ideas of the Social Studies of Science and Technology will be introduced in order to comprehend how the contextualization process directly affects the object of study. Second, it will be noted that this object has an emerging ontological status since the relationships that compose it must be constantly redefined. Third, Marilyn Strathern's contributions to the previous debates will be highlighted through her reflections on the scales. Finally, through the concepts of operational-logic, make-scale and mereology it will be sustained that ontology, epistemology, methodology and reflexivity are in permanent feedback. In addition, it will be emphasized that this link implies reevaluating the political position of the researcher, the way in which fieldwork and the non-human world are understood.
\end{abstract}

\title{
KEY WORDS
}

Context, ontology, reflexivity, Social Studies in Science and Technology, Marilyn Strathern.

Lo que observamos no es la naturaleza misma, sino la naturaleza expuesta a nuestro método de interrogación - Werner Heisenberg

\section{INTRODUCCIÓN: LA PREGUNTA POR LA REFLEXIVIDAD}

Los marcos investigativos se componen de forma general por un conjunto sistemático de conceptos, instrumentos y procedimientos que se articulan lógicamente para elaborar un conocimiento científico sobre la realidad. Evaluar la rigurosidad de estos marcos implica recalibrar continuamente sus criterios de validez, generalidad y objetividad. Por este motivo hacer ciencia pasa ineludiblemente por problematizar y actualizar los fundamentos de su labor.

La autoevaluación científica comúnmente comienza con dos interrogantes centrales: $i$ los marcos investigativos permiten analizar de forma neutra al objeto de estudio o por el contrario la sistematicidad de sus datos es un efecto artificial generado por estos?, ¿la ontología (¿qué existe?), la epistemología (¿cómo conozco?), la metodología (¿por medio de que procedimientos se puede acceder el objeto?) y la reflexividad (vigilancia de la subjetividad del investigador) se diferencian y concatenan linealmente como etapas investigativas o se entrelazan y afectan recíprocamente?

Los debates de la fenomenología, el psicoanálisis, el feminismo, los estudios en ciencias, el postcolonialismo y la antropología han confrontado el ideal de 
"neutralidad valorativa" como base de la objetividad científica al alertar que los investigadores no pueden llegar a ser observadores imparciales de la realidad. Sus preguntas, conocimientos, experiencias y relaciones de poder mediarían directamente en la forma en que conoce y representa el mundo, por lo que la rigurosidad de la ciencia no consistiría en dejar de lado la subjetividad sino en visibilizarla de forma crítica y propositiva. Ampliando estas ideas, Strathern (1990) advierte que los investigadores pueden creer que acceden al fenómeno que estudian al identifican una conectividad inmanente en los datos que lo integran, pero tras esta supuesta transparencia los marcos analíticos podrían ser confundidos con el objeto de estudio forzando una conexión entre sus elementos. En este sentido, las metáforas usadas por los antropólogos:

Campos comunicacionales, ecosistema, formación social, inclusive estructura, todos ellos construyen contextos globales para la interconexión de eventos y relaciones. Su peligro radica en hacer aparecer al sistema como el sujeto bajo escrutinio en vez del método de escrutinio. Los fenómenos llegan a aparecer contenidos o abarcados por la sistémica, y por lo tanto como sistémicos (p. 7) ${ }^{1}$.

Si bien los modelos teóricos no reflejan la realidad en sí misma, puesto que son abstracciones que la simplifican en orden de comprenderla, al momento de analizar los datos obtenidos por medio de conceptos estos antes de operar como herramientas descriptivas se pueden convertir en dispositivos prescriptivos. Es decir, en vez de identificar y seguir relaciones, pre-ordenarían invisiblemente los datos y excluirían los que no encajan en su contexto lógico. A razón lo de lo expuesto, si las metáforas que integran los marcos investigativos se toman como neutras se puede crear o exagerar la sistematicidad del objeto estudiado. Por ejemplo, en el caso de la ecología Serje (1999) expone que sus conceptos básicos se han basado en analogías de otras disciplinas tales como: estrategia, red, autorregulación, energía, productores/consumidores, entre otros, para construir sus modelos explicativos, por lo que:

El análisis de las analogías entre las colectividades humanas y los fenómenos ecológicos pone también en evidencia el hecho de que para la construcción de modelos explicativos y predictivos, se parte de representaciones de la naturaleza que hacen parte de los procesos sociales y políticos, en el marco de los cuales estos se gestan (p.47)2.

Por consiguiente, la ecología no es simplemente una ciencia que tiene un acceso directo y privilegiado a la naturaleza ya que sus categorías son parte de un horizonte histórico de posibilidad que incentiva y limita lo pensable y lo impensable. Por este tipo de mediaciones es que Wagner (1981) denuncia los ses-

1 Todas las traducciones del inglés son elaboraciones propias.

2 Por ejemplo, la Selección Natural no es un concepto ajeno de su contexto histórico-político. Según Heredia (2011) existe un amplio conjunto de premisas ontológicas compartidas entre Adam Smith sobre la sociedad de mercado y Charles Darwin sobre la economía de la naturaleza. Esto evidencia una estrecha relación entre estos postulados políticos y biológicos la cual permite afirmar que el darwinismo es un tipo de liberalismo biológico. 
gos de las investigaciones antropológicas: "Lo que el trabajo de campo inventa, por lo tanto, es su propio entendimiento; las analogías que crea son extensiones de sus propias nociones y las de su cultura" (p. 12). Bajo la misma denuncia, Briones (2007) expone críticamente que las teorías sobre la identidad pueden terminar siendo normativas porque se naturalizan acríticamente como referentes interpretativos y morales de los cambios sociales: "la idea de que las identidades pueden ser flexibles, fragmentadas, múltiples, construidas, contrastivas deviene convicción de que las identidades deben ser todo eso" (p.75)

$\mathrm{Si}$ el investigador supone la trasparencia de sus conceptos puede terminar extrapolando relaciones particulares y artificiales de conectividad ante la especificidad y heterogeneidad de los datos en los que luego percibe patrones generales y naturales. Lo expuesto implica que los principios y los medios a través de los cuales se entiende la realidad ordenan el mundo que se percibe, lo cual conduce a reconocer que la ontología, la epistemología, la metodología y la reflexividad se encuentran de alguna forma interconectados. Para comprender este complejo vínculo el presente escrito sintetiza, evalúa y amplia críticamente los debates contemporáneos sobre el ejercicio de contextualización con la finalidad de identificar los nuevos desafíos y posibilidades que acarrea la investigación social. Es esta medida busca interrogar: ¿el contexto preexiste al objeto de estudio?, ¿cómo debe de ser entendido el estatus ontológico del objeto?, ¿se debe abandonar la idea de contexto para trazar redes o es necesario identificar y contrastar múltiples contextos por medio de escalas?, y, por último, ¿cuáles son las implicaciones de las ideas anteriores para la labor investigativa?

Para responder las preguntas anteriores, primero, se introducirán las ideas centrales de los Estudios Sociales de la Ciencia y la Tecnología con la finalidad de dimensionar cómo el proceso de contextualización afecta directamente el objeto de estudio. Segundo, se señalará que este objeto posee un estatus ontológico emergente puesto que se deben redefinir constantemente las relaciones que lo componen. Tercero, se resaltarán las contribuciones de Marilyn Strathern a los debates anteriores por medio de sus reflexiones sobre las escalas. Por último, mediante los conceptos de lógica-perceptiva, hacer-escala y mereología se sustentará que la ontología, la epistemología, la metodología y la reflexividad se encuentran en una retroalimentación permanente. Además, se resaltará que este vínculo implica reevaluar la posición política del investigador, la forma en que entiende el trabajo de campo y el mundo no-humano.

\section{ESTUDIOS SOCIALES DE LA CIENCIA Y LA TECNOLOGÍA}

El contexto se ha concebido en las ciencias sociales como el marco objetivo y preexistente en el que está posicionado y circunscripto el objeto de estudio, por lo que sus cualidades solo pueden tener sentido y ser comprendidas en relación con las demás entidades que lo integran. De allí una de las máximas de la antropología: "colocar las cosas en contexto". Por ejemplo, el concepto de cultura 
operó como una totalidad lógica que permitía explicar la vida social puesto que desde sus orígenes esta disciplina consideró que:

las ideas occidentales sobre el ser individual eran inadecuadas, incluso equivocadas, y que los sistemas religiosos, las funciones sociales, las estructuras de parentesco y los modos de producción suponen que el análisis debía comenzar al nivel de la sociedad o cultura como un todo (Otto \& Bubant, 2010, p. 8)

Pero para los investigadores contemporáneos las totalidades lógicas como naturaleza, cultura o sociedad son insuficientes para comprender el desbordamiento de relaciones temporales, geográficas, políticas, económicas, ambientales, artefactuales, etc. que integran sus objetos de estudio. Tal es así que Tsing (2010) reconoce que los antropólogos ya no tienen certeza sobre cuáles son los "contextos apropiados" para delimitar sus investigaciones. Como respuesta directa a la denominada "crisis del contexto" se encuentran los Estudios Sociales de la Ciencia y la Tecnología (ESCT) una de las corrientes teóricas actuales más potentes que se ha embarcado en la tarea de abandonar la división entre mundo social y mundo material.

Los ESCT son un campo académico heterogéneo que se congrega en la crítica a la consideración de que habitamos un mundo pasivo y pre-existente. Por el contrario, postulan que la realidad social seria el producto emergente de la negociación activa entre agentes humanos y no-humanos. Para sustentar estas ideas toman a la ciencia como objeto de estudio por excelencia para sustentar que esta se encontraría influenciada no solo por los marcos morales, políticos y epistemológicos de sus investigadores, sino también por los artefactos mediante las cuales configuran y elaboran la identidad de los hechos científicos. De este modo, los objetos antes de simplemente reflejar o condensar pasivamente conocimientos y voluntades humanas serian entidades que las limitarían, estabilizarían y potenciarían puesto que poseerían capacidad de agencia (Asda \& Mose 2012, Callon 1986, 2008, Latour 1995, 2005, 2007, Law 2004, Mol 2004, Woolgar \& Lezaun 2013).

Para esta corriente teórica el mundo se encuentra en formación permanente debido a que la existencia de los objetos antes de ser dada es enactuada, es decir, es producto la práctica permanente de interacción entre mediaciones humanas y no-humanas. En esa medida, los objetos para Woolgar \& Lezaun (2013) "son traídos en existencia, son realizados en el curso de una determinada actividad práctica" (p. 323). Es decir, son imaginados, articulados y estabilizados por medio de los procedimientos mediante los cuales se conocen. Por ello en su estudio sobre bolsas de basura afirman que la identidad de lo representado es en parte formada por la organización del texto que las nombra en el marco de un contexto específico. Así, por ejemplo:

La mundanidad de la bolsa de basura no es pre-dada; esta en cambio se logra en virtud de su articulación como parte de la estructura del orden moral del que forma parte (...) Entonces lo qué la cosa es, para lo qué sirve, lo que debe contener, y lo que es un comportamiento (in)apropiado a través de esta 
están todos ligados a (y ejemplifica) una estructura del orden moral (p.332).

La forma de texto que representa el objeto parte de las premisas básicas sobre lo que cuenta como realidad para el investigador, en este sentido: "Una declaración sobre el mundo también es (...) una declaración sobre los motivos de la persona que hace la declaración" (Law, 2004, p. 98). Por lo tanto, el orden, los contrastes, los énfasis narrativos, junto con las entidades y las relaciones que se nombran, no describirían transparentemente una realidad externa, sino que mediarían la posibilidad de percibir y dar cuenta de lo que se estudia porque la organización del escrito encarna su andamiaje conceptual. A causa de esto:

La invocación, construcción y constitución del 'contexto' están íntimamente implicadas en la determinación situada de lo que es el objeto. En otras palabras, la construcción e invocación de «el contexto» resulta ser importante para el logro del carácter ontológico de las entidades en juego (Woolgar \& Lezaun, 2013, p. 327).

Otro ejemplo del entrelazamiento entre los marcos investigativos y el mundo material es el de la fermentación de ácido láctico desarrollada por Louis Pasteur. Latour (1995) al estudiar si este ácido fue un descubrimiento o una invención paradójicamente propone abandonar dicho interrogante. Este antropólogo afirma que no hay fuerzas físicas que se agrupan en un fenómeno que luego un científico como Pasteur podría identificar transparentemente (descubierto inductivamente); ni tampoco que un fenómeno físico es simplemente moldeado e interpretado libremente bajo unas condiciones históricas particulares (construido deductivamente). Por el contrario, existirían acontecimientos que transforman las trayectorias de las entidades estudiadas y de los que las estudian, por lo que habría una invención-descubrimiento de éstas:

Pese a sus dudas, Pasteur no dictó a los hechos como debían hablar. Se mezcló con ellos y compartió con ellos su historia, su cuerpo, su laboratorio y la asamblea de sus colegas, ofreciéndoles otra oportunidad. Los descubría tanto como los moldeaba. Todos se coaligan en esta ocasión, materia y forma de los unos y de los otros (...) No existe ser alguno, como el fermento láctico o la gravitación universal, que "desborde" sus condiciones históricas de emergencia -lo cual no equivale a decir, sin embargo, que solo sea el resultado del trabajo humano. Precisamente lo que ya no podemos hacer es elegir entre esas dos versiones (1995, p. 106).

Los conceptos, las preguntas y los estilos argumentativos junto con los artefactos tecnológicos, las rutinas y los espacios, afectarían en lo que podemos considerar como existente porque influirían en la representación y estabilización de la identidad del objeto. Como ejemplo de ello Callon (2008) analiza la forma en que las ciencias económicas performatizan la economía. Según este académico los debates teóricos actuales no pueden explicar satisfactoriamente las agencias calculadoras, el por qué se toman decisiones económicas, puesto que se limitan a entender las acciones de los sujetos como precondicionadas a estructuras mentales, marcos simbólicos o redes sociales. Bajo una consideración crítica, propone que este comportamiento estaría directamente influenciado por los mismos me- 
dios a partir de los cuales las personas conocen y se posicionan en el mercado: los dispositivos de cálculo con los que cuentan.

Las herramientas contables no describirían pasivamente una realidad económica externa, sino que operarían como medios que delimitan los tipos de agentes y de relaciones existentes, por lo que guiarían la comprensión, la organización, la legitimación y la proyección de las agencias calculadoras. Esto implica que las decisiones económicas no se podrían explicar simplemente a partir de cualidades naturales, procesos de aculturación o las redes de conexión, sino que se requiere tomar en cuenta el mundo artefactual a partir del cual se interactúa con el mercado. Debido a ello el homo economicus si existiría, pero no como una condición humana, sino como un anudamiento de lugares y temporalidades especificas en el cual el sujeto es: "formateado, enmarcado y equipado con prótesis que le ayudan en sus cálculos y que son, por lo general, producidas por las ciencias económicas" (p. 62).

El comportamiento económico surge por lo tanto de la elaboración y la comprensión de un contexto por medio de dispositivos de cálculo creados en las ciencias económicas, esto implica que el conocimiento académico sobre el mundo y los medios para alcanzarlo (métodos y dispositivos) afectan activamente el mundo conocido. El estudio de la configuración activa de la ontología del objeto por parte del proceso investigativo es por tanto la estrategia de reflexividad que proponen los ESCT la cual permite evidenciar que "los métodos en las ciencias naturales y las ciencias sociales difícilmente captan su propia performatividad" (Law, 2004, p. 122). En otras palabras, el investigador debe objetivar cómo los medios mediante los cuales conoce afectan lo conocido, pero no solo tomando en cuenta elementos discursivos y teóricos, sino también los artefactos materiales que sostienen e integran su mundo social.

\section{ESTATUS ONTOLÓGICO DEL OBJETO}

La investigación antropológica contemporánea ya no busca partir de los grandes conceptos holistas (naturaleza, cultura, sociedad, estructura) como se hacía en los periodos anteriores puesto que por medio de estos se delimitaba de antemano el contexto explicativo de su objeto de estudio. Por el contrario, los debates actuales sostienen que su objeto no posee un estatus ontológico dado, sino que este es emergente a causa de su dinamismo, es decir, de las múltiples relaciones contingentes que entrelaza (espaciales, temporales, ecológicas, materiales, etc.), por lo que debe ser redefinido constantemente (Hastrup, 2005).

La falencia de los abordajes holistas es que suponen de antemano el estatus ontológico del objeto limitando así sus múltiples posibilidades existenciales, de allí que han: "confundido lo que deben explicar con la explicación" (Latour, 2005 , p. 8), como advertía previamente Strathern en el caso de las metáforas. Por esta razón la preocupación de Viveiros de Castro (2015) sobre la falta de sensibilidad e innovación investigativa es legítima: 
Cuando un chamán te muestra una flecha mágica extraída de un hombre enfermo, un médium es poseído por un dios, un hechicero construye laboriosamente una muñeca vudú, solo vemos una cosa: sociedad (creencia, poder, fetichismo). En otras palabras, solo nos vemos a nosotros mismos (p.12).

Los nuevos debates antropológicos proponen captar la identidad contingente, abierta, interconectada y particular del objeto de estudio. Por este motivo las categorías, antes de ser entendidas como medios descriptivos de un mundo pre-existente, se toman como recipientes aglutinadores de relaciones contextuales y dinámicas. Debido a ello Latour (2005) sostiene que: “'grupo', 'actor', 'agencia', 'traducción' y 'fluido'. Al igual que la noción de red, no designan lo que se está mapeando, sino cómo es posible mapear cualquier cosa" (p. 174). En vista de lo anterior, la investigación científica se comprende como un proceso de cartografiar la especificidad de las relaciones que condensan la identidad del objeto y le dan un valor diferencial en el mundo. Tsing (2015) proporciona un claro ejemplo de este tipo de estrategias:

Si las categorías son inestables, debemos verlas emerger dentro de los encuentros. Utilizar nombres de categorías debe ser un compromiso para rastrear los ensamblajes en los que estas categorías obtienen un sostén momentáneo. Solo desde aquí puedo volver a encontrarme con los Mien y los matsutake en un bosque de cascadas. ¿Qué significa ser «Mien» o ser «bosque»? Estas identidades entraron en nuestra reunión a partir de las historias de la ruina transformadora, incluso cuando las nuevas colaboraciones las cambiaron (p. 29)

Prejuzgar el objeto, es decir, delimitar de antemano las lógicas que lo constituyen, termina por exagerar su sistematicidad y de este modo se oculta su dinamismo, heterogeneidad y desplazamiento. Considerando esta problemática, Law (2004) expone abiertamente que su objeto antes de encontrarse delimitado y ser estático se movía y transformaba su identidad a través de múltiples prácticas y lugares: "el objeto de estudio y sus contextos estaban moviéndose continuamente. En consecuencia, no solo encontramos que estábamos cambiando entre diferentes enfermedades hepáticas alcohólicas, pero también, y con inquietud, entre diferentes problemas" (p. 78). Law describe su confusión en los siguientes términos:

Inicialmente fuimos invitados a explorar el diagnóstico y el tratamiento de la enfermedad hepática alcohólica. Llamemos esto objeto número uno. Pero a medida que avanzamos en el estudio y entrevistamos a los profesionales descubrimos que a menudo hablábamos de enfermedad hepática (objeto número dos) en lugar de la enfermedad hepática alcohólica. O, más específicamente, estábamos discutiendo la cirrosis alcohólica (número tres). $\mathrm{O}$, muy comúnmente, la charla era del abuso del alcohol y sus implicaciones para las personas y el sistema de atención de la salud (cuatro). O (no necesariamente lo mismo) era del alcoholismo (cinco). $\mathrm{O}$ (como nosotros acabamos de ver para el caso del Dr. Nixon) podría tratarse de la calidad de vida en general en relación con el abuso de sustancias (seis). El problema, entonces, era cómo pensar este desplazamiento: el hecho de que el objeto de estudio parecía escaparse y deslizarse de una entrevista a la siguiente (p. 78). 
Del mismo modo Mol (2002) encuentra que la arterioesclerosis no es una enfermedad delimitada y enteramente preexistente a su estudio, sino que la interrelación de la vida cotidiana de los pacientes, los procedimientos médicos y artefactos clínicos enactúan diferentes enfermedades, de allí que "No es que haya dos, cinco o setenta variantes de la aterosclerosis, sino que hay multiplicidad" (p.51). En esta medida, la existencia del objeto no está dada por fuerzas internas o externas, sino que se gesta por medio de su entrelazamiento con el mundo. Su dinamismo y movilidad yace en que no se puede separar de las relaciones que lo componen puesto que: "los contextos tienden a venir en plural, trabajando para atraer cosas juntas en formas potencialmente conflictivas y superpuestas" (Asda \& Moser, 2012, p. 303).

La identidad de lo estudiado no se debe presuponer como punto de partida teórico, sino que es el punto de llegada contingente de la investigación porque el objeto no posee un estatus existencial totalmente fijo, por consiguiente, "'Materialidad', tanto como 'contexto' y sus términos afines, deben entenderse como el resultado contingente de las prácticas, en lugar de una la realidad fundamental a ser iluminada por una investigación ontológica" (Woolgar \& Lezaun, 2013, p. 327). En vista de lo expuesto, los ESCT proponen como principio metodológico "seguir los actores mismos". Se deben rastrear las acciones y relaciones que objeto enlaza y transforma para reconocer que las condiciones que rodean su existencia no son una barrera para acceder a el, sino el tejido mismo que le da una identidad, consistencia y fuerza en la realidad.

Debido a lo anterior, Latour (2005) argumenta que se debe abandonar la idea de "contexto" para poder identificar "redes". Por su parte, el contexto pretende aislar unos principios generales y básicos que posibilitan y limitan de forma causalista y antropocéntrica la acción, intensión y forma de los seres involucrados. En cambio, en la red se busca trazar un mapa plano que visibilice el ensamblaje interactivo y simétrico entre humanos y no-humanos reconociendo que estas relaciones no son unidireccionales en un sentido causalista, sino que cada entidad influye en las trayectorias de las demás, por lo que poseen agencia.

La propuesta de Latour pretende abandonar las distinciones micro/macro, interacciones/estructuras y local/global para ver cada entidad como producto de redes más o menos conectadas. Por ello, "cualquier interacción dada parece desbordarse con elementos que ya están en la situación provenientes de algún otro tiempo, de otro lugar, y generados por alguna otra agencia" (2005, p. 166). De allí que para evitar proyecciones antropomórficas se deban ver las acciones no como efectos de la conciencia o marcos sociales, sino como nodos en los que confluyen y median fuerzas y agentes heterogéneos que no se pueden suponer a priori.

En síntesis, para este antropólogo explicar es "trazar una red" con múltiples mediadores de modo que "nos daríamos cuenta que no hay ya necesidad de una sociedad que esté 'detrás"” (2005, p. 108). De este modo, Mialet (2012) siguiendo la propuesta de los ESCT desarrolló el concepto de "sujeto centrado-distribuido" para estudiar las redes que conformaron al cosmólogo Stephen Hawking. Esto le permitió exponer que las personas no son una entidad delimitada en un 
cuerpo que produce conocimiento en su reflexión mental, sino el producto de las múltiples materialidades que amplían, estabilizan, filtran, limitan, extienden y afectan su identidad, acción y pensamiento. Por ello su tarea investigativa consistió en:

describir las diferentes materialidades (las máquinas, los asistentes, el propio Hawking, los estudiantes, los colegas, los diagramas, los periodistas, los artículos, las películas y los libros, mi propia presencia, los archiveros, los artistas, la arquitectura de la ciudad, los archivos, la estatua) que constituyen a Stephen Hawking -esto es, constituyen su presencia (p. 9)

La propuesta de los Estudios Sociales de la Ciencia y la Tecnología es sumamente valiosa para la investigación social puesto que invita a no prejuzgar la identidad del objeto de estudio. Considerar la emergencia ontológica del objeto permite alcanzar el extrañamiento necesario para que el investigador amplié su sensibilidad en campo y problematice su propio sentido común disciplinar. Ahora bien, antes de tomar acríticamente los aportes de los ESCT el siguiente apartado evaluará en las limitaciones de sus postulados al preguntar ¿se debe abandonar la idea de contexto como sugiere Latour?, ¿cuáles son las falencias comparativas de los ESCT?, ¿qué otro aporte ofrece la antropología para entender la multiplicidad ontológica del objeto?

\section{REDES VS ESCALAS}

Los ESCT exponen que colocar las cosas en contexto, es decir, circunscribirlas dentro de una totalidad explicativa, antes de permitir acceder al fenómeno bajo estudio, puede terminar por filtrar y moldear los datos de campo para que entren en su estructura lógica. Por este motivo, si se utilizan categorías preestablecidas para explican nuevos fenómenos no se captan sus especificidades ontológicas, sino que se proyectan los principios teóricos en su percepción. En consecuencia, los marcos investigativos no solo delimitan lo pensable, sino que también producen "acuerdos con implicaciones políticas. Organiza arreglos y reuniones de cosas -y reporta los arreglos de esas cosas- que podrían haber sido de otro modo" (Law, 2004, p. 143).

Pero rastrear las redes que constituyen al objeto de estudio no implica necesariamente abandonar la construcción de un contexto como sostiene Latour porque se sacrificaría la posibilidad de realizar comparaciones en la investigación. Por ejemplo, considero que Law (2004) califica la realidad como difusa, indefinida, elusiva y efímera debido a que no cruza diferentes contextos para darle sentido a los datos que encuentra, por este motivo niega la posibilidad de comparación a razón de que solo puede identificar en estas articulaciones inestables:

ya no hay cualquier forma general de moverse sin esfuerzo de un lugar a otro sin atender a las especificidades. No hay un mundo general y no hay reglas generales (...) La idea de la transportabilidad universal del conocimiento universal siempre fue una quimera (2004, p. 135) 
De forma crítica a los ESCT Tsing (2010) argumenta que se necesitan yuxtaponer contextos heterogéneos para comparar y comprender los datos puesto que las figuraciones de los actores requieren estudiar "los contextos que hacen para sí mismos y de los cuales están hechos" (p.62). Por esta razón critica que el estudio de Callon (1986) sobre las Vieiras en Francia siguió a los actores humanos y nohumanos involucrados, pero no contrastó los datos con contextos exteriores que podrían explicar mejor el fenómeno, en este caso, el cultivo de estas ostras por parte de científicos japoneses.

La evaluación anterior de Tsing a los ESCT está basada en los postulados de Marilyn Strathern. Para esta última antropóloga un fenómeno no es una entidad auto-contenida, es decir, una totalidad unificada y preexistente que articula de forma coherente elementos discretos, sino un tejido heterogéneo, incompleto y parcialmente conectado mediante diferentes escalas con su propia especificidad y complejidad. En este sentido, también se comparte la idea de multiplicidad ontológica al ver las cosas como relaciones, pero cada entidad es producto de relaciones que tienen sentido mediante otras relaciones (Strathern 1988, 1999, 2004).

Si bien para Strathern el contexto conecta y da sentido a las cosas, este no localiza al objeto diferenciándolo de otras relaciones y delimitando relaciones internas y externas. Por el contrario, dichas fronteras se confrontan puesto que el investigador debe contextualizar las premisas investigativas que guían su ejercicio de descripción para contrastarlas con los múltiples contextos estudiados para evidenciar sus resonancias, especificidades y límites explicativos. De este modo, se generan formas de conocimiento innovadoras ya que la investigación seria esencialmente un ejercicio comparativo que capta la multiplicidad de su objeto.

Debido a lo anterior, para Morita (2013) «la etnografía Stratherniana transforma reflexivamente su propio método al formar relaciones mecánicas con la otra perspectiva, las relaciones internas de la etnografía también se refieren a las conexiones que el etnógrafo hizo en el campo" (p. 212). En otras palabras, la estrategia de Strathern realiza una conexión parcial entre perspectivas heterogéneas que se retroalimentan y operan como una extensión de las otras, pero que nunca agotan su particularidad fusionándose. De allí que en su trabajo en Melanesia esta antropóloga considere que las personas igual que las cosas son producto de relaciones que yuxtaponen múltiples contextos, por lo que, por ejemplo, el género permite clasificar y es producto de relaciones externas al género, los sujetos se equivalen o diferencian como personas, espíritus o canoas dependiendo de la perspectiva del observador

La falencia de la teoría de Latour para Morita (2013) yace en que carece de reflexividad al momento empírico de seguir conectores y trazar sus asociaciones inmediatas, a diferencia de Strathern ya que para esta antropóloga "la contextualización juega un papel fundamental en la mediación de lo conceptual y lo empírico en la etnografía" (p. 217). De hecho, para Strathern, la dificultad de trazar redes consiste en córtalas, en otras palabras, al seguir actores no hay marcadores claros para identificar donde se detienen sus asociaciones para así establecer límites en su análisis (Strathern 1996). En sintonía con las críticas 
señaladas, Rocheleau (2011) propone trasformar el trazado de red de Latour en modelos policéntricos:

incorporar las distintas posiciones y perspectivas de múltiples grupos de personas y varias especies y ensamblajes de plantas y animales, junto con artefactos, tecnologías y elementos físicos de su entorno. No es solo cuestión de acercarse, de obtener la única historia verdadera. Se trata de «conseguirla" a través de los ojos de una diversidad de actores en distintas posiciones, en redes de actores complejos (p. 215).

No es suficiente con seguir las trayectorias de los actores mismos para mapear las relaciones que ensamblan, sino que a su vez se deben contrastar los múltiples puntos de vista de los actores involucrados en el fenómeno estudiado, es decir, yuxtaponer contextos. Este fue el desafío que afrontó Tsing (2005) al encontrar una amplia multiplicidad de discursos de la naturaleza en Indonesia: "Los activistas de Banjarmasin, Yakarta y Manggur cuentan diferentes historias del bosque: el bosque es un lugar salvaje desafiante; el bosque es un sitio de resistencia popular y gestión comunal; el bosque es el medio de redes sociales" (p. 262).

Pero estas diferencias sobre un espacio y lucha ambiental no fueron un impedimento investigativo para Tsing debido a que le permitieron conocer la historia territorial y las relaciones de poder desde diferentes perspectivas y agencias entrelazadas y en choque. Por ello comprendió que era necesario elaborar estrategias conceptuales-y-metodológicas para estudiar los momentos y los medios a partir de los cuales estas historias diferenciales se articulaban y lograban condensarse en la imagen de un objeto común. En este sentido, identificó el anudamiento de su objeto de estudio en múltiples relaciones y escalas espaciales:

Los individuos, incluidos científicos, políticos y activistas, aplican sus perspectivas eclécticas en la formación de proyectos de creación-de-la-naturaleza. No tiene sentido buscar el conocimiento ambiental singular «indonesio» o uno que divida claramente en grupos étnicos o religiones. En cambio, podríamos comenzar identificando confluencias distintivas de conocimiento, así como los nodos de práctica y discurso informados por estas confluencias (Tsing, 2005, p. 113).

Por lo tanto, los sujetos desarrollan sus creencias-y-prácticas en medio de la articulación entre contextos heterogéneos, lo cual permite explicar que "La fuerza personal de la 'naturaleza' y su apariencia como objeto de reflexión dependen tanto de esta localización como de su naturaleza cosmopolita" (2005, p.153). La valoración y representación de la naturaleza surge en medio de la especificidad local de relaciones humanas y no-humanas que ensambla y los múltiples contextos globales con los que se interconectan dichas relaciones.

De igual modo, el conocimiento local sobre el ambiente no está anclado a un territorio delimitado, sino que se elabora por medio de experiencias, resignificaciones, préstamos y traducciones, por consiguiente: "Las «comunidades» están constituidas en relación con otros tipos de proyectos de hacer-escala, incluidas las burocracias, las naciones y los organismos internacionales de poder y exper- 
ticia" (2005, p. 264). Valga resaltar que el entrelazamiento de contextos es un proceso de hacer-escala que resulta ser trascendental para el antropólogo porque la da sentido a sus experiencias de campo. Hastrup (2013) expone que en una conversación en el Ártico con una anciana esta no describía su pasado, sino que las diferentes preguntas, intensiones y momentos permitían contextualizar las experiencias vividas:

Conversando con ella era como si un mundo entero se estuviera desplegando en frente mío, y yo no pudiera más que sentirme inmensamente agradecida por su tiempo y su historia - y por ser una antropóloga (...) Realmente, la vida de la mujer era un todo visto desde su perspectiva, y cuando era relatado; pero su historia era estimulada por mi propia presencia y con mucha comprensión retrospectiva. En consecuencia, la sustancia de la conversación trascendió vastamente el tiempo y el espacio de nuestra reunión y testificó al punto de que era ella quien producía el espacio, el tiempo y la escala relevante (p. 151).

La entrevista misma operaba como un marco interpretativo emergente que permitía analizar en retrospectiva sucesos bajo diferentes puntos de vista, por lo que operaba como una escala que le daba sentido a sus recuerdos de objetos, paisajes y personas. A razón de lo expuesto, no hay una sistematicidad clara en las acciones, interpretaciones y creencias de los sujetos ya que como sostiene Haraway (1991) "La topografía de la subjetividad es multi-dimensional" (p. 331) puesto que esta consiste en "conexiones parciales".

Por último, si bien los ESCT poseen diversas falencias que limitan el ejercicio comparativo estas se pueden eludir por medio los aportes del pensamiento escalar de Strathern. Por ello la investigación de Tsing (2015) es paradigmática para la antropología contemporánea pues logra articular las estrategias de redes y de escalas. Por un lado, sigue a los actores mismos identificando el ensamblaje de relaciones humanas y no-humanas, en este caso el crecimiento en bosques de los hongos Matsutake en ecologías compartidas con otros seres. Por el otro, yuxtapone de forma escalar diferentes contextos y actores (bosques de Estados Unidos, compañías de comercio, tiendas de venta en Japón) para identificar sus interconexiones y especificidades. Esta estrategia le permitió a Tsing entender con una sorprendente productividad analítica las complejas lógicas que guían las cadenas capitalistas de acumulación que crean valor de relaciones no-capitalistas.

Para condensar lo expuesto hasta el momento, un gran aprendizaje de los debates investigativos contemporáneos es que para mapear las relaciones emergentes que integran al objeto de estudio no podemos dar por sentado su contexto (redes) pero a su vez debemos yuxtaponer relaciones externas a este para darle sentido a sus datos (escalas). Queda por preguntar ¿de qué forma es posible entender y representar la retroalimentación existente entre los marcos investigativos y el objeto de estudio?, ¿cómo este vínculo conduce a repensar la posición política del investigador, el trabajo de campo y el mundo no-humano? 


\section{REFLEXIVIDAD POST-CONTEXTUAL}

La relación entre la ontología, la epistemología, la metodología y la reflexividad en la investigación científica se puede entender como una concatenación de procedimientos separados. El problema de dicha linealidad es que da por sentada la existencia y estabilidad del objeto de estudio dentro de un contexto lo cual termina por desincentivar el mapeo permanente de las múltiples relaciones que lo componen y, en consecuencia, genera una ilusión de que este no es afectado por el proceso investigativo. Además, a causa de este orden, puede surgir una desproporcionalidad entre dichos procedimientos, puesto que inconscientemente los investigadores terminan por darle un sobreénfasis a la comprobación teórica lo cual conduce a proyectar una falsa sistematicidad en los datos estudiados (Ver Gráfica 1.).

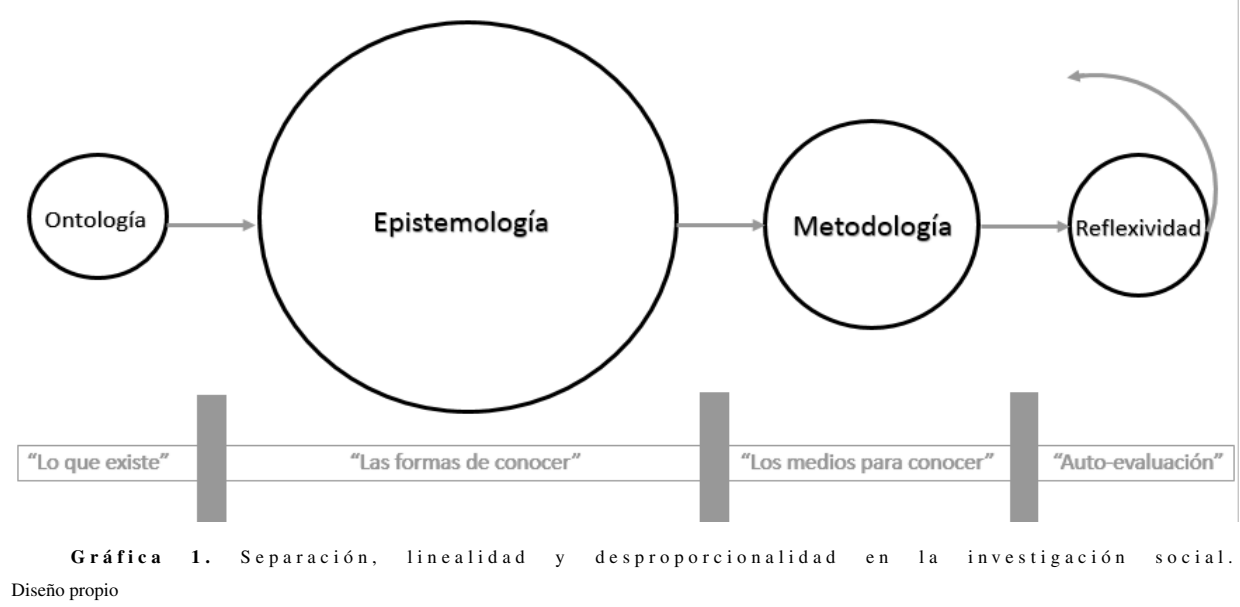

No obstante, si se toman los aprendizajes complementarios sobre redes y escalas, estos cuatro procedimientos se deben entender como yuxtapuestos y en retroalimentación, por lo que requieren la misma relevancia en las investigaciones. A continuación, se estudiará cómo el acceso y la expresión del objeto se encuentra mediada por una influencia recíproca entre tres elementos: la lógicaperceptiva, el hacer-escala y la mereología (Ver Gráfica 2.).

\section{- Lógica-perceptiva}

Si bien la epistemología opera como un sistema de conceptos abstractos para identificar relaciones en el mundo, simultáneamente nos dice cuáles son las re-laciones relevantes, de este modo proporciona focos metodológicos de análisis. En otras palabras, una teoría no es totalmente abstracta como para encontrarse enteramente desligada de un contexto perceptivo, esta comúnmente posee unos lineamientos prácticos (aunque no siempre sean explícitos) los cuales señalan qué elementos y relaciones del mundo pueden ser significativas y cómo acceder a ellas por medio de instrumentos específicos. 
A su vez, cada metodología no es solamente un mecanismo pasivo de recolección de información puesto que una estrategia metodológica siempre encarna un contexto lógico que le permite conceptualizar lo que cuenta o no como dato y relación. Por consiguiente, aunque ambos sean procedimientos diferentes con sus propias complejidades, la epistemología y la metodología se encuentran constitutivamente entrelazadas por una lógica-perceptiva. En pocas palabras, la aplicación y el análisis de los datos por medio de un instrumento refleja sus supuestos teóricos.

Así, por ejemplo, el perspectivismo multinaturalista de Viveiros de Castro (2010) no es simplemente un marco de conceptos y lógicas abstractas que conceptualiza los puntos de vista de seres que se consideran como humanos, sino que también opera como una metodología que busca encontrar similitudes internas en la diversidad de datos etnográficos en la amazonia y en occidente para ver cómo el primer modelo impugna políticamente el segundo. La teoría del actor-red elaborada por Latour (2005) tampoco es solo un marco epistemológico para conceptualizar el mundo no-humano, puesto que simultáneamente es un dispositivo metodológico que pretende analizar los datos de campo rompiendo la división mundo social/mundo material. En esta medida, el primer modelo opone frontalmente los datos y el segundo los entrelaza lateralmente, epistemología y metodología se articulan como estrategias comparativas.

\section{- Mereología}

La conexión entre la epistemología y la ontología se establece a través de un contexto lógico que posibilita o limita la conceptualización del objeto de estudio. Cada epistemología se sostiene bajo una teoría causal sobre la realidad que opera postulando (o problematizando) la existencia y articulación de un Todo (piénsese en naturaleza, sociedad, historia, cultura, capitalismo, comunidad, etc.) y sus partes (sujeto, discurso, gen, práctica, etc.). Por consiguiente, la mereología, es el esqueleto lógico detrás de cada concepto, el cual le permite ver, agrupar, omitir, separar y explicar los vínculos posibles en el mundo.

Existen diferentes mereologías en las que: 1. El todo es más que la suma de las partes, por lo que como sistema general las trasciende y ordena (Piénsese en el Funcionalismo, el Estructural-funcionalismo y el Estructuralismo). 2. Las partes son autónomas ya que no logra constituirse una totalidad (el Interaccionismo, la Etnometodología, la Deconstrucción, el Interpretativismo). 3. El todo y las partes se afectan mutuamente (la Teoría de sistemas, el Estructural-constructivismo, el Postfeminismo, el Postcolonialismo y el Posestructuralismo). 4. No existe ni el todo ni las partes, una entidad no está aislada ni condicionada por un sistema externo y preexistente, puesto que se encuentra desbordada por múltiples relaciones y contextos (Debates ontológicos contemporáneos: Haraway 1995, Halbmayer 2012, Strathern 2004, Latour 2005, Hanks \& Severi 2014, Tsing 2015) (Perdomo 2020). 


\section{- Hacer-escala}

La ontología y la metodología se encuentran interrelacionadas a partir de la escala de análisis de la que se parta. El punto de vista (piénsese en lo económico, lo histórico, lo global, etc.) que se adopten en los estudios directamente limitan y posibilitan el acceso a los datos y por consiguiente condiciona la percepción del objeto de estudio y su expresión ontológica. En esta medida la escala es un contexto perceptivo a partir del cual diferentes estrategias e instrumentos filtran la información del campo marcando qué relaciones se deben valorar, conectar y excluir.

La anisotropía es un claro ejemplo para pensar en términos de hacer-escala. Este concepto señala que la medición de las propiedades de los cristales varía dependiendo de la dirección en que se analizan. De la misma manera sucede en la antropología, por ejemplo, los estudios sobre las etnicidades contemporáneas cuando se centran en escalas locales muestran que las concepciones y las prácticas socio-ambientales de los pueblos indígenas impugnan la epistemología moderna, por lo que sus identidades se ven como medios de resistencia. De modo opuesto, al concentrarse en escalas estatales tienden a ver sus expresiones sociales moldeadas en forma de respuesta a normatividades políticas, por lo que las identidades tienden a caracterizarse como parcialmente limitadas por los constreñimientos institucionales. En cambio, las investigaciones centradas en escalas globales muestran los medios a partir de los cuales los grupos étnicos se pliegan a discursos internacionales para ser favorecidos en términos políticos y económicos, de este modo las identidades se analizan como capitales estratégicos.

De acuerdo a la perspectiva a análisis se pueden obtener respuestas diferentes del mismo objeto, pero ninguna puede agotarlo porque, por ejemplo, como exponen De la Cadena \& Starn (2009): "las prácticas culturales, las instituciones y la política indígenas se hacen indígenas en articulación con lo que no se considera indígena en la formación social particular en la que existen" (p. 196). Cada escala necesita de las relaciones de las demás escalas para existir, en esa medida las investigaciones actuales buscan yuxtaponer perspectivas para reconocer la multiplicidad y complejidad de su objeto (Strathern 2004, Hastrup 2012, Tsing 2005, 2015, Perdomo 2019). 


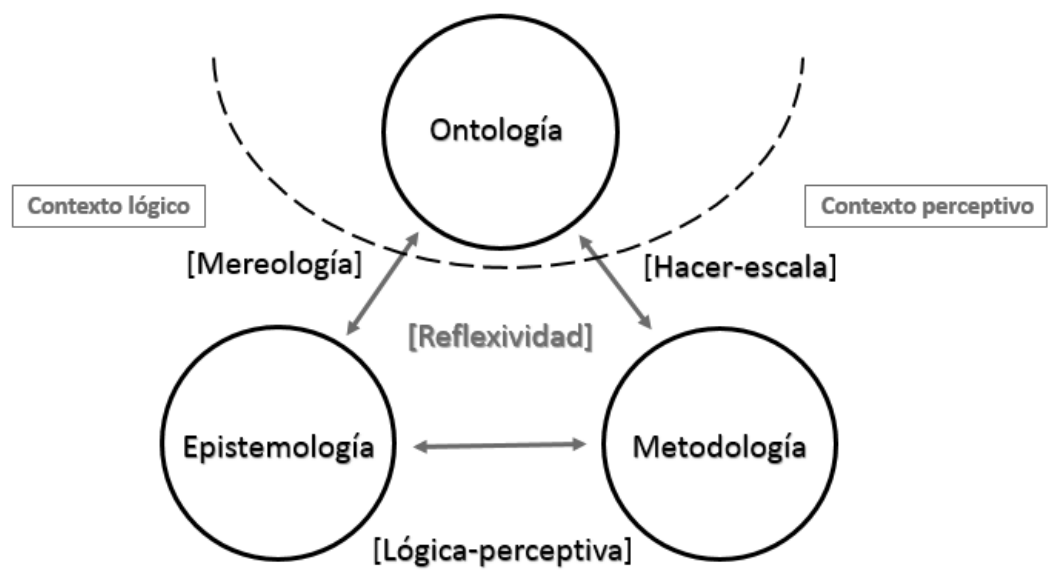

Gráfica 2. Retroalimentación y proporcionalidad en los procedimientos investigativos.

Diseño propio.

Las ideas desarrolladas hasta el momento señalan que la metodología y la epistemología se encuentran entretejidas con la ontología del objeto de estudio. Estos argumentos pueden ser tomados erróneamente como remanentes de un constructivismo radical. Pero de hecho parten de la consideración de que la existencia del objeto de estudio es independiente del investigador, por ello en la segunda gráfica la ontología aparece rodeada por una línea punteada que la separa de los otros dos momentos. Aun si, el punto a resaltar es que la capacidad de expresión de este objeto y la posibilidad de acceder a él siempre se encuentra mediada, es decir, limitada e incentivada, por la mereología y la escala, la primera entendida como el contexto lógico y la segunda como el contexto perceptivo. En consecuencia, la epistemología y la metodología no solo conceptualizan-yrecolectan información, sino que también co-producen los datos.

Ahora es momento de especificar que la reflexividad, la cual se encuentra en el centro de la gráfica anterior, requiere ser entendida como el ejercicio de vigilancia de la interrelación entre la ontología, la epistemología y la metodología. Creer que cada uno es totalmente autónomo ante el otro genera la ilusión de que el objeto este preexiste enteramente a la investigación y que los medios de conocerlo no influyen en éste, sino que lo describen transparentemente. De este modo se puede generar una falsa separación entre el mundo y el investigador en nombre de la objetividad. Pero como se expuso a lo largo de este escrito, la realidad conocida se encuentra enlazada con los medios a partir de los cuales accedemos a ésta. A continuación, dicho reconocimiento implicará reevaluar: la posición política del investigador, la comprensión del trabajo de campo y la relevancia del mundo no-humano en el análisis social. 


\subsection{Posición política del investigador}

La retroalimentación de los marcos investigativos con el objeto no conlleva a que el investigador se encuentre totalmente aprisionado por los sesgos de sus métodos, por lo que su conocimiento solo sería una falsa proyección intelectual. Al contrario, es necesario trasgredir estos relativismos al reconocer que la reflexión sobre la condición subjetiva del conocimiento no es su impedimento práctico, sino un garante de su misma objetividad (Haraway, 1995). En la antropología la objetividad investigativa se alcanza al encontrar un punto de simetría entre los procedimientos investigativos y las experiencias de campo, es decir, al colocarlos en un mismo nivel de diálogo y de vigilancia para eliminar jerarquías y sobreénfasis.

Esta simetría es productiva analíticamente a razón de que, por un lado, permite reconocer que, si bien cada momento posee su propia complejidad, no se pueden desligar totalmente las etapas de análisis y de escritura puesto que se encuentran parcialmente imbricadas. Por el otro, incentiva el desarrollo de habilidades de atención para suspender los juicios teóricos y políticos ante la riqueza significativa de los datos, y de este modo se limita la proyección anticipada de premisas en el objeto de estudio.

Para alcanzar esta simetría investigativa primero es necesario confrontar el tipo de metáfora a partir de cual se relacionan los marcos investigativos con las experiencias de campo. Un marco investigativo tiende a comprenderse comúnmente como una especie de lente que se lleva a campo para observar e interpretar las lógicas del mundo que se esconden detrás los discursos, las prácticas, los objetos y demás representaciones. Pero este marco debería ser mejor entendido como un conjunto reflexivo de preguntas abiertas que pueden dar indicios generales sobre las relaciones que componen la realidad. Por consiguiente, para utilizar metáforas más productivas, es posible pensar un marco teórico-metodológico no tanto como un lente que filtra el mundo, sino más bien una brújula que ofrece pistas ante la complejidad del campo en el que se encuentra inmerso el investigador.

No se puede explorar a ciegas el campo sin un marco teórico-metodológico guía, es decir, sin conceptos-instrumentos que sugieran posibles caminos de conectividad al pretender otorgarle una primacía inductiva a la experiencia (como pretendía la Teoría Fundamentada de Glaser \& Strauss). Pero a su vez, tampoco es suficiente darle un centramiento deductivo a estos marcos ya que es necesario reconocer que los conceptos-e-instrumentos que acompañan en el proceso investigativo no descubren y describen la realidad tal cual es. Ello se debe a que la abstracción y generalidad de las herramientas científicas hace que estas sean permanentemente desbordadas por el dinamismo, heterogeneidad, sistematicidad parcial y especificidad de los datos, por lo que en sí mismas resultan ser insuficientes para explorar el mundo.

Por ejemplo, el caso más problemático sobre la ilusión de transparencia en las teorías se encuentra en el uso de tipologías. Gnecco \& Langebaek (2016) sostienen que esto sucede en la arqueología debido a que la radicalización tipo- 
lógica ha conducido a generalizar patrones prestablecidos excluyendo la innovación explicativa y a omitir el dinamismo temporal y geográfico del registro arqueológico por medio de modelos estáticos. Ante estos errores, que vuelven a la arqueología "tiránica", proponen que las tipologías deberían ser dinamizadas a partir de una perspectiva crítica, heurística y no prescriptiva:

crítica porque la actitud complaciente frente a su tiranía se reemplaza por una actitud militante contra ella; heurística porque se usan como medios para alcanzar interpretaciones alternativas y sugerentes y no como destinos últimos y definitivos; y no prescriptiva porque en vez usarlas como hilos que se deben seguir se usan como partes constitutivas de tramas complejas y conectivas (p. $\mathrm{X})$

Lo anterior sugiere que los marcos investigativos, en este caso bajo las tipologías, necesariamente deben ser recalibrados y problematizados por la sensibilidad y creatividad etnográfica. En este tipo de tipo de desafíos, Tsing (2005) autocríticamente se dio cuenta de la incompletud de sus categorizaciones formales para explicar el activismo medioambiental en Indonesia:

Los linajes contingentes que he descrito constituyen el marco social para el amor hacia la naturaleza, pero estos no expresan la fuerza del sentimiento de amor a la naturaleza. No nos dicen cómo y por qué las personas particulares son movilizadas por el romance de la naturaleza. Esto requiere preguntar a los individuos, cuyas trayectorias personales funcionan dentro de la confluencia de los linajes culturales, pero hacen algo personalmente significativo de estos (p. 147).

Tsing usó recursivamente las historias de vida de cuatro individuos para dimensionar las potencias afectivas y las circunstancias existenciales que condujeron a estos actores hacia una comprensión de la naturaleza y una agencia especifica en el activismo, lo cual le permitió comprender que el ambientalismo global: "solo puede existir en los diálogos y la sobreposiciones entre estas preocupaciones distintivas acerca de la naturaleza" (2005, p. 153). Es decir, el cruce de escalas es el sostén de las creencias y los afectos por lo que su estudio requería de la articulación simétrica entre lo conceptual y lo empírico.

El ejemplo anterior evidencia que es posible colocar los marcos investigativos y los datos de campo en un mismo nivel. La metáfora más clara de este ejercicio sería la de una conversación en la que se gesta un diálogo abierto a partir del cual el uno está atento del otro. Si hay una paridad se contaminan mutuamente pues surgen resonancias a partir de las cuales, por un lado, los datos resignifican la teoría-y-la-metodología al enriquecer y problematizar el significado y la aplicabilidad de las categorías de análisis, y, por el otro, estos marcos influirían en los datos al otorgarles una mayor robustez expresiva que revelaría la interconexión y la especificidad del objeto de estudio.

Para que los juicios teórico-morales no sean un obstáculo para alcanzar la simetría investigativa es necesario cultivar una apertura sensible hacia las formas en las que el objeto de estudio emerge, permitiendo así que "el campo nos hable". En otras palabras, que las experiencias de campo cobren valor no solo 
por estar encasilladas en los marcos investigativos, sino a su vez por la capacidad de reflexividad del investigador. Explorando en este tipo de debates Grimson (Badaró, Carozzi \& Otros, 2011) afirma:

creo que nuestros sufrimientos y nuestras preguntas (o la de aquellos que nos rodean) tienen que seguir marcando nuestras agendas de investigación y nuestras preguntas, pero no tienen que marcar nuestras respuestas. Porque si no contribuimos poco a ampliar la imaginación, a poder pensar nuevas cosas, mirar desde lugares diferentes. Porque desde el sufrimiento hay cosas que no se ven (p. 296).

En este punto se cruzan los marcos investigativos del investigador con sus juicios éticos, ¿se debe tomar una postura política en los estudios científicos?, ¿los investigadores deben representar estratégicamente las luchas sociales?, ¿las explicaciones saturadas de matices pueden entorpecer procesos políticos? (Badaró, Carozzi \& Otros, 2011). Para poder analizar esta compleja tensión se debe comprender primero la naturaleza misma de la práctica antropológica. Ingold (2017) afirma que la función de esta disciplina es dialogar con el mundo, por lo que no estudia al otro, sino con el otro para comparar críticamente nuestras múltiples posibilidades de existencia, en consecuencia:

debemos exigir el derecho de hablar con nuestras propias voces, y decir lo que pensamos sobre la base de nuestras investigaciones, independientemente de si concuerda con el pensamiento de nuestros interlocutores. Podemos haber aprendido de lo que nos han dicho, pero como antropólogos en lugar de etnógrafos, no es nuestro trabajo reflejarlo. Al igual que los practicantes de cualquier otra disciplina, debemos estar listos para hablar con nuestras propias voces y no escondernos detrás de las voces de los demás. Si fallamos en hacerlo, nos veremos a excluidos de los grandes debates de nuestro tiempo -debates acerca de cómo deberíamos vivir- o descubriríamos que somos convocados solo para proporcionar el material para que otros nos manipulen a su voluntad (p. 24)

Los antropólogos necesitan identificar matices y contrastar perspectivas, mostrar choques internos, en suma, visibilizar la contingencia y la tensión de toda formación social. Para Tsing (2005) es "política y también intelectualmente útil ofrecer un análisis simétrico de la fricción tanto en la industria de extracción de recursos como en su oposición" (p.221) puesto que permite ver las potencialidades y las fragilidades, tanto en los procesos de enajenación y violencia como en los de democratización para abandonar las creencias en la generalidad de la dominación y los llamados naturales a la resistencia. De este modo es posible

${ }^{3}$ Comúnmente en la antropología se toma a la proximidad con los demás como un ideal ético que conduce a un trabajo comprometido. Complejizando esta idea, Willerslev (2006) sostiene que: "la proximidad sería imposible en la ausencia de distancia porque entonces el experienciador y la experiencia se fundirían, se convertirían en uno, por lo tanto, haciendo la experiencia del otro imposible. Por consiguiente, lo que la antropología está buscando no es una experiencia de verdadera proximidad radical, sino un tipo de experiencia que nos coloque en contacto con los otros y aun así nos separe de ellos, manteniéndonos a distancia" (p. 40). 
vislumbrar que el mundo se encuentra en construcción permanente, por lo que la historia se encuentra abierta a múltiples caminos (Tsing, 2015).

\subsection{Trabajo del campo}

La recontextualización permanente del objeto de estudio confronta la comprensión de qué es el campo y cuál es la duración necesaria en este para realizar una investigación. Ello se debe a que, primero, implica reconocer que en el proceso de investigación no se va a campo como si este fuera un contexto delimitado y estático, una totalidad sistémica a conocer. Por el contrario, como señala Jaramillo (2013) se hace campo debido a que este consiste en "un conjunto de trayectorias que interconectan lugares, tiempos, personas y objetos" (p. 15). En este sentido, el campo para la antropología se elabora al trazar relaciones que problematicen los contextos dados (Hastrup, 2013).

Segundo, en consecuencia, el trabajo de campo intensivo deja de ser suficiente para realizar este mapeo activo de las relaciones que constituyen el objeto de estudio, puesto que, si este no preexiste enteramente, sino que solo lo podemos comprender a partir de sus redes y escalas: "Tener material etnográfico 'suficiente' no es una simple función de larga duración, sino más de las preguntas formuladas" (Hastrup, 2013, p.152). Mayor tiempo puede enriquecer un estudio, pero no asegura obligatoriamente un mejor acceso al conocimiento si no hay reflexividad sobre los marcos investigativos. Por consiguiente, las cualidades más relevantes de la investigación social no son la vastedad de tiempo y de datos, como se sostenía en las investigaciones modernistas, las investigaciones contemporáneas demandan la suspensión del juicio, la sensibilidad y la creatividad etnográfica, por ello Strathern argumenta: "Si uno puede realizarle 'grandes' preguntas a datos 'pequeños' entonces la diferencia entre lo grande y lo pequeño desaparece" (Strathern, 2004, p. XX).

\subsection{Entidades no-humanas}

Si bien desde el realismo se considera que habitamos un mundo dado y pasivo del que estamos separados, esto no es totalmente diferente del constructivismo radical, pues este, desde la misma premisa antropocéntrica, sostiene que el mundo no posee un sentido en sí mismo, sino que la agencia y la significación es ejercida exclusivamente por los humanos. Contrario a estas posturas, los debates de la antropología contemporánea confrontan la contextualización antropocéntrica del mundo al argumentar que es necesario estudiar las influencias y entrelazamientos entre seres humanos y no-humanos para comprender la realidad social. Por esta razón Tsing (2005) denuncia:

Nos encontramos atrapamos en las literaturas sobre símbolos y significados, sobre clase y colonialismo, sobre la mercantilización y la penetración 
del capitalismo. Pero estas literaturas hacen poco para ayudarnos a entender la naturaleza: su diversidad, su poder y sus constreñimientos, o sus formas multifacéticas de entrar en las historias humanas (...) Los biólogos de la conservación segregan a los no humanos; los ecólogos políticos los toman con demasiada frecuencia como recursos para el uso humano (p. 173)

Debido a los sesgos anteriores en las investigaciones científicas esta antropóloga propone realizar una "descripción crítica" que considere a los seres humanos no como los actores centrales en la naturaleza, sino uno más entre diversas fuerzas históricas. Por este motivo, resalta la necesidad de articular la etnografía y la historia natural en una comprensión ampliada del mundo que reconozca que: "los humanos y otras especies entran en las formas de la vida a través de redes de relaciones sociales" (2013, p.28). De este modo, se debe descentrar el papel del ser humano en el ambiente para identificar que seres heterogéneos confluyen y moldean directamente los mundos multi-especie que habitamos y el mundopor-venir (Tsing, 2015).

A pesar de este tipo de llamados Kohn (2013) indica que a la antropología le cuesta reconocer la agencia de entidades no-humanas puesto que se naturaliza acríticamente la idea de que el lenguaje y la representación son lo mismo, "incluso cuando no utilizamos explícitamente el lenguaje o lo simbólico en nuestras herramientas teóricas" (p.39). Esto conlleva erróneamente a que se considere que el contexto etnográfico solo existe como referente simbólico que nos constituye como humanos, por lo que los hechos sociales solo tendrían sentido en relación con este. Pero dicha concepción omite que:

No se aplica por completo en dominios como las relaciones humanoanimales que no están completamente circunscriptas por lo simbólico, pero que son sin embargo semióticas. Los tipos de modalidades representacionales compartidas por todas las formas de vida -modalidades que son icónicas e indexadas- no dependen del contexto de la misma manera que las modalidades simbólicas (2013, p. 39).

Retomando la teoría semiótica de Charles Peirce, Kohn sustenta que la capacidad de representación no se limita a lo simbólico y, por lo tanto, lo humano. Por esta razón expone que seres vivos no-humanos entran en el juego activo de la significación al existir otras formas de conocimiento interactivo con el mundo mediante las cuales se comunican y realizan asociaciones y predicciones. De hecho, en medio de sus heterogeneidades y críticas mutuas, para los debates antropológicos contemporáneos es imperativo problematizar la división cultura/naturaleza, puesto que la percepción y acción humana tampoco estaría condicionada por símbolos (o genes), sino que, más allá de estos, nuestro conocimiento sobre el mundo surgiría en la práctica activa y el ensamble parcial con agencias y significaciones de seres no-humanos (Latour 2005, Ingold 2000; Hanks \& Severi 2014; Hastrup 2012; Henare, Holbraad, \& Wastell 2007; Kohn 2013; Rocheleau 2011; Severi 2011, Tsing 2015). 


\section{CONCLUSIONES}

La contextualización ya no es el ejercicio de certidumbre que delimita y explica al objeto de estudio, sino una práctica de experimentación que requiere el abandono del sobreénfasis teórico para abrirse a la posibilidad de mapear redes y escalas en las que existe el objeto. Para abordar este complejo desafío analítico el presente escrito comenzó exponiendo que el investigador no posee una posición neutra para acceder a la realidad dado que se encuentra inmerso en las relaciones que estudia. En esta medida, las analogías científicas son el horizonte que median su posibilidad de imaginar y conocer la realidad.

En el primer apartado, se sintetizaron las ideas centrales de los Estudios Sociales de la Ciencia y la Tecnología. Principalmente se identificó la performatividad de los métodos de investigación pues se argumentó que no se puede separar el proceso de conocer, siempre mediado por entidades no-humanas, de lo conocido en la medida en que los contextos no se encuentran "yaciendo pasivamente 'allá afuera' esperando a ser descubiertos" (Asda \& Moser, 2012, p. 303), sino que su elaboración influencia la ontología del objeto.

Segundo, se expuso que el objeto de estudio posee un estatus ontológico emergente ya que es producto de múltiples relaciones, de allí que para los ESCT estos postulados conduzcan a abandonar el contexto para trazar redes como contrapropuesta. El contexto no explicaría el objeto porque da por sentado lo que existe en cambio la red no la supone a priori, sino que más bien busca visibilizar su despliegue particular y contingente. Tercero, se criticaron los postulados de Latour con base en las ideas de Strathern, Tsing Rocheleau y Hastrup sobre las escalas para al final resaltar la importancia de complementar ambas estrategias investigativas. De este modo, se resaltó la importancia del ejercicio de yuxtaponer perspectivas para darle sentido a los datos mientras se sigue el ensamblaje de relaciones humanas y no-humanas.

Por último, como crítica a la separación, linealidad y desproporcionalidad entre la ontología, la epistemología, la metodología y la reflexividad se identificó su entrelazamiento y necesidad de simetría por medio de los conceptos de mereología, hacer-escala y lógica-perceptiva. En este punto, la reflexividad sería entendida como el proceso de reconocimiento vigilante dicha interrelación, la cual implica que el investigador debe suspender su juicio, que el campo no es un espacio sino una práctica y que es necesario integrar el mundo no-humano en la investigación antropológica.

Las reflexiones meta-teóricas anteriores sobre el contexto evidencian las dificultades del proceso de delimitación del objeto de estudio, más no su imposibilidad. La delimitación debe ser entendida como el proceso de captar simultáneamente la especificidad e interconexión de objeto para establecer una posición desde la cual comprender el mundo. Pero para esta tarea no hay métodos universales, sino experimentos situados que solo son posibles y efectivos si primero, como en este escrito, se parte de su problematización.

En suma, este texto se elaboró para resaltar la necesidad de ser críticos e innovadores ante los procedimientos naturalizados en la investigación social que 
pueden predisponer los datos a encontrar. Por ello se resaltó la importancia de conversar simétricamente con y a partir del mundo mientras reflexionamos sobre los medios a partir de los cuales lo conocemos. Nutrir los marcos investigativos capitalizando la riqueza significativa de la experiencia misma permite alcanzar una apertura reflexiva y sensible hacia nuestro objeto de estudio. Esta apertura permite mapear las múltiples relaciones que lo entretejen sin apresurarnos a ver el contexto como un marco objetivo preexistente que lo fija y le da sentido, pero tampoco como un impedimento que debemos abandonar a causa de que ocultaría su desborde de relaciones. Por el contrario, la conversación investigativa posibilita que el contexto sea entendido como un intermediador dinámico a partir del cual nos damos a la oportunidad de pensar en las múltiples posibilidades existenciales de nuestro objeto.

Las ideas desglosadas en este artículo nos invitan a abandonar el centramiento excesivo en el marco teórico para poder ver su entrelazamiento con la metodología, la reflexividad, la ontología, el sujeto que conoce y el mundo mismo. Debo reconocer que dicho entrelazamiento tiende a tornase un laberinto en el recorrido investigativo. Pero solo podemos estudiar un mundo complejo si valoramos la incertidumbre y la curiosidad como cualidades científicas que, a partir de la búsqueda de asombro, nos obligan a problematizar los ejercicios de contextualización.

\section{BIBLIOGRAFÍA}

ASDA, K. \& MOSE, I. (2012): Experiments in Context and Contexting. Science, Technology, \& Human Values 37, 4, pp. 291-306

BADARO, M., CAROZZI, M., ESCOBAR, A., FONSECA, C., GRIMSON, A., SEMÁN, P., WILDE, P. (2011): Conversaciones sobre la diferencia. Encuentro con Arturo Escobar. Tabula Rasa, No. 15, pp. 275-298

BRIONES, C. (2007): Teorías performativas de la identidad y performatividad de las teorías. Tabula Rasa, pp. 55-83

CALLON, M. (1986): "Some elements of a sociology of translation: domestication of the scallops and the fishermen of St Brieuc Bay". Power, action and belief: a new sociology of knowledge? pp.196-223. London: Routledge.

(2008): "Los mercados y la performatividad de las ciencias económicas", Apuntes de investigación, No. 14, pp. 11-68

DE LA CADENA, M. \& STARN, O. (2009): Indigeneidad: problemáticas, experiencias y agendas en el nuevo milenio. Tabula Rasa, No 10, pp. 191-223

GNECCO, C. \& LANGEBAEK, C. (2016): “Contra la tiranía del pensamiento tipológico”, En: Cristóbal Gnecco \& Carl Henrik Fangebaek (Eds.) Contra la tiranía tipológica en arqueología: Una visión desde Suramérica, Colombia, Ediciones Uniandes, pp. IX-XIV

HALBMAYER, E. (2012): Amerindian mereology: Animism, analogy, and the multiverse. Indiana, 29, pp. 103-125

HANKS, W. \& SEVERI, C. (2014): Translating worlds. The epistemological space of translation. Hau: Journal of Ethnographic Theory, 4, 2, pp. 1-16 
HARAWAY, D. (1991): “Conocimientos Situados: la cuestión científica en el feminismo y el privilegio de la perspectiva parcial". En: Ciencia, Cyborgs, Mujeres. La reinvención de la Naturaleza, pp. 313-346. España, Ediciones Cátedra

HASTRUP, K. (2005): Social anthropology. Towards a pragmatic enlightenment?. Social Anthropology, 13, 2, pp. 133-149

(2012): Scales of attention in fieldwork: Global connections and local concerns in the Arctic. Ethnography, 14, 2, pp. 145 - 164

HEREDIA, J. (2011): Etología animal, ontología y biopolítica en Jakob von Uexküll. Filosofia e História da Biología, Vol. 6. No. 1, pp. 69-86

HENARE, A.; HOLBRAAD, M. \& WASTELL, S. (2007): "Introduction: Thinking through things", En Amiria Henare, Martin Holbraad and Sari Wastell (Eds.). Thinking through things. Theorising artefacts ethnographically, New York, Routledge, pp. 1-31

INGOLD, T. (2000): The Perception of the Environment. Essays on livelihood, dwelling and skill, London: Routledge

(2017): Anthropology contra ethnography. Hau: Journal of Ethnographic Theory, 7, 1, pp. 21-26

JARAMILLO, P. (2013): Etnografías en transición: Escalas, procesos y composiciones. Revista Antípoda, No 16, pp. 13-22

KOHN, E. (2013) How forests think. Toward an anthropology beyond the human. Los Angeles: University of California Press

LATOUR, B. (1995): ¿Tienen historia los objetos? El encuentro de Pasteur y de Whitehead en un baño de ácido láctico. Isegoría, No 12, pp. 92-109

(2005): Reassembling the Social. An Introduction to Actor-NetworkTheory. New York, Oxford University Press.

(2007): Nunca fuimos modernos: Ensayos de antropología simétrica, Argentina Siglo XXI Editores

LAW, J. (2004): After Method. Mess in social science research, EE.UU, Routledge

MIALET, H. (2012): Introduction. En: Hawking Incorporated: Stephen Hawking and the Anthropology of the Knowing Subject, pp. 1-9. University of Chicago Press: Chicago

MOL, A. (2002): Body multiple. Ontology en medical practice. Duke University Press. United States of America.

MORITA, A. (2013): The Ethnographic Machine: Experimenting with Context and Comparison in Strathernian Ethnography. Science, Technology, \& Human Values, 39(2): 214-235.

PERDOMO MARÍN, J. (2019): "Bebiendo los cabellos de Dios. Aproximaciones teórico-metodológicas a las ceremonias de yagé en Colombia”. Boletín de Antropología, 34 (57):147-173.

(2020): Agencias, mundos y ontologías como escenarios de problematización de la antropología contemporánea. Maguaré, Vol 33 No. 2. (Próximo a publicar)

ROCHELEAU, D. (2011): "Rooted Networks, Webs of Relation, and the Power of Situated Science: Bringing the Models Back Down to Earth in Zambran". Mara J. Goldman, Paul Nadasdy \& Matthew D. Turner (Eds.) Knowing Nature: Conversations at the Intersection of Political Ecology and Science Studies, 209-226. Chicago: University of Chicago Press.

SERJE, M. (1999): La concepción naturalista de la naturaleza. Un desafío al ambientalismo. Revista de Antropología y Arqueología, Vol. 11, No. 1-2, pp. 5-70 
SEVERI, C. (2011): The arts of memory Comparative perspectives on a mental artifact. HAU: Journal of Ethnographic Theory, 2, 2, pp. 451-485

STRATHERN, M. (1988). The gender or the gift. University of California press. Los Angeles

(1996): Cutting the network. The Journal of the Royal Anthropological Institute, 2(3), pp. 517- 535 . (1999): “The Ethnographic Effect I", En Property, Substance and Effect: Anthropological Essays on Persons and Things, London, The Athlone Press (2004): Partial Connections, Updated Edition, Walnut Creek, Altamira Press

TSING, A. (2005): Friction: An Ethnography of Global Connection. Pricenton University Press, United States of America.

(2010): "Worlding the Matsutake Diaspora: Or, Can Actor-Network Theory Experiment With Holism?" En: Ton Otto \& Nils Bubandt (Eds.) Experiments in Holism, Malaysia: Blackwell, pp. 47-66

(2013): "More-than-Human Sociality: A Call for Critical Description", Kirsten Hastrup (Ed.) Anthropology and Nature, New York, Routledge, pp. 27-42

(2015): The mushroom at the end of the world. On the Possibility of Life in Capitalist Ruins, United Kingdom, University Press

VIVEIROS DE CASTRO, E. (2010): Metafísicas Caníbales: Líneas de antropología postestructural, Buenos Aires: Katz Editores (2015): "Who is Afraid of the Ontological Wolf? Some Comments on an Ongoing Anthropological. The Cambridge Journal of Anthropology, 33(1), pp. 2-17.

WILLERSLEV, R. (2006) "To have the World at a Distance: Rethinking the Significance of Vision for Social Anthropology", En Grasseni, C. (Ed.), Skilled Visions: Between Apprenticeship and Standards, pp. 24- 46. The EASA Series: Learning Fields, vol. 6, Berghahn Books: New York.

WAGNER, R. (1981): "The assumption of culture". En: The invention of culture, Chicago, University of chicago press, pp. 1-16

WOOLGAR, S. \& LEZAUN, J. (2013): The wrong bin bag: A turn to ontology in science and technology studies? Social Studies of Science, 43(3), pp. 321-340 